\title{
REVISÃo DE LITERATURA Evolução do DSM quanto ao critério categorial de diagnóstico para o distúrbio da personalidade antissocial
}

\author{
DSM evolution as categorical diagnostic criterion for the antisocial \\ personality disorders
}

Marco Antônio Silva Alvarenga', Carmen E. Flores-Mendoza², Daniel Foschetti Gontijo ${ }^{3}$

\section{RESUMO}

Objetivo: Realizar um breve percurso sobre o desenvolvimento conceitual de um dos construtos psicológicos de maior evidência nos dias atuais, a saber: o transtorno de personalidade antissocial (TPAS). Especificamente, esse percurso se realiza no sistema categórico proposto pela Associação Americana de Psiquiatria (APA), o Manual Diagnóstico e Estatístico de Distúrbios Mentais (DSM). Método: Utilizou-se a revisão literária sobre a evolução e a avaliação do construto associada a pesquisas empíricas consultadas nos principais livros e periódicos de reconhecimento internacional na área, tais como: Personality and Individual Differences, Psychological Medicine, Annual Review of Clinical Psychology, Psychological Bulletin, Journal of Abnormal Psychology, Journal of Personality Assessment, International Journal of Offender Therapy and Comparative Criminology, Aggression and Violent Behavior, Handbook of Psychopathy, entre outros. Resultado: Observa-se que o diagnóstico do TPAS é baseado nos critérios categóricos e não dimensionais. Isso significa que o sistema não consegue predizer a priori a variabilidade (intensidade) dos traços desse transtorno por ser o DSM desenvolvido no reconhecimento de sintomas e síndromes. Conclusão: Apesar de o TPAS ter passado por diversas revisões e de apresentar insuficiência taxonômica, ele ainda é amplamente utilizado no diagnóstico e no prognóstico clínico de condições relacionadas ao comportamento social desviante.

\section{ABSTRACT}

Objective: This present work does a brief developmental route about one of the most evidence contemporary construct: the antisocial personality disorder (APD). Specifically, this guide is realized in accordance to categorical system raised by American Psychiatry Association (APA), the Diagnosis Statistical of Mental Disorders (DSM). Method: This article uses a literature revision about the evolution and assessment of the construct associate to empirical studies counseled in main books and journals worldwide recognized in the area as: Personality and Individual Di-

\footnotetext{
1 Universidade Federal de Minas Gerais (UFMG), Universidade Fumec - Fundação Mineira de Educação e Cultura, Faculdade de Ciências Humanas (FCH), Departamento de Psicologia. em Neurociências.

3 Universidade Fumec - Fundação Mineira de Educação e Cultura, Faculdade de Ciências Humanas, Departamento de Psicologia.

Endereço para correspondência: Marco Antônio Silva Alvarenga

Av. Antônio Carlos, 6627, Departamento de Psicologia, sala 4046, LADI

UFMG - 31270-901 - Belo Horizonte, MG

Telefone: (31) 3409-6275

E-mail: alvarenga.mas@gmail.com
} 


\section{Keywords}

Personality disorders, antisocial personality disorder, DSM, categorical and dimensional diagnostic system. fferences, Psychological Medicine, Annual Review of Clinical Psychology, Psychological Bulletin, Journal of Abnormal Psychology, Journal of Personality Assessment, International Journal of Offender Therapy and Comparative Criminology, Aggression and Violent Behavior, Handbook of Psychopathy, among others. Result: It observes that diagnosis of APD is based in categorical criteria not dimensionally. It means that system doesn't predict to prior the traits variability (intensity) of that disorder because the DSM it was development to recognize symptoms and syndromes. Conclusion: Despite the APD has gone for different revisions and still presents taxonomic deficient it remains widely used in clinical diagnoses and prognoses related to social deviant behavior.

\section{INTRODUÇÃO}

Ao longo da história médica e psiquiátrica surgiram vários termos para designar os padrões não adaptativos das dimensões afetivas, sociais e do comportamento. As nomenclaturas diagnósticas, assim como as descrições sintomáticas, mudavam de autor para autor. Essas diferenças eram salientadas pelas percepções sociais e culturais que cada estudioso tinha do fenômeno em uma dada época, influenciando o modo de descrever e tratar um indivíduo com desajustamento desses padrões ${ }^{1,2,3}$.

A difusão de critérios para a formulação diagnóstica não era econômica e muito menos precisa. Isso significa que a divergência entre especialistas para classificar um quadro clínico dificultava o delineamento de um plano de intervenção bem como um o prognóstico eficaz. Essa foi uma das premissas que levaram a Associação Americana de Psiquiatria (APA) e a Organização Mundial da Saúde (OMS) a desenvolverem critérios mais parcimoniosos e consensuais para diagnosticar os distúrbios mentais, entre os quais estão os Transtornos de Personalidade. O Manual Diagnóstico e Estatístico de Distúrbios Mentais (DSM) e o sistema de Classificação Internacional de Doenças (CID) foram criados, respectivamente, pela APA e a OMS, em uma tentativa de catalogar uma série de doenças mentais identificadas, clinicamente descritas e sistematizadas ${ }^{4,5}$

O grande avanço da Psiquiatria e da Psicologia Clínica propiciou várias pesquisas sobre os transtornos mentais a partir da década de 1970, especialmente o TPAS 6 . Apesar de haver certa dificuldade para determinar quais seriam os sintomas e as diretrizes diagnósticas desses tais transtornos? estudos foram desenvolvidos para avaliar os critérios e a validade diagnóstica do DSM e do CID, bem como estabelecer quais seriam as melhores categorias para identificar os transtornos de personalidade e tratá-los adequadamente $2,8,9$.

Quer as diretrizes diagnósticas sejam precisas ou nem tanto, um volume considerável de investigações foi realizado utilizando os critérios diagnósticos do DSM para reconhecer os padrões patológicos da personalidade. Entre tais padrões disfuncionais, em decorrência do grande impacto social imediato, o TPAS - e sua coocorrência com a psicopatia (PP) - vem sendo estudado sistematicamente por vários pesquisadores muito antes da origem desse manual, mas sendo extensamente investigado a partir dele $e^{1,10}$. Há o reconhecimento de que a dificuldade de adaptação às expectativas sociais, a irresponsabilidade, a impulsividade, a instabilidade no trabalho, a agressão sem motivos aparentes, o abuso de substâncias, entre outros sintomas, causam grande prejuízo à sociedade e aos próprios indivíduos que produzem esses comportamentos. Comparando-se esses sintomas aos critérios diagnósticos dos manuais, observase que estes últimos reportam mais classes comportamentais do que afetivas, tornando difícil a identificação de uma outra condição clínica, considerada pelo CID e pelo DSM como equivalente ao TPAS, qual seja a PP11-15.

Apesar da utilização de manuais como referências para formular o diagnóstico dos transtornos de personalidade, o DSM é indiscutivelmente um dos grandes sistemas de classificação utilizado por pesquisadores de diversos países junto com o CID-10 ${ }^{16}$. Nesse sentido, esta seção contemplará o estudo do DSM, cujos critérios costumam guiar as investigações do TPAS e as diferenças individuais da PP. Como o DSM da APA é amplamente utilizado no mundo ${ }^{17}$, por isso traça um panorama evolutivo no que se refere ao diagnóstico dos distúrbios de personalidade desviantes, permitirá verificar como se compreenderam os padrões antissociais ao longo do tempo.

\section{MÉTODO}

Para a confecção deste ensaio teórico, utilizaram-se os manuais da Associação de Psiquiatria Americana junto com textos de autores especializados sobre o uso do DSM e do CID-10 no reconhecimento do TAPS. Também se realizou uma busca de estudos teóricos e empíricos sobre tal transtorno em jornais científicos como: Personality and Individual Differences (fator de impacto igual a 1,598), Psychological Medicine (fator de impacto igual a 4.718), Annual Review of Clinical Psychology (fator de impacto igual a 2,235), Psychological 
Bulletin (fator de impacto igual a 12,568), Journal of Abnormal Psychology (fator de impacto igual a 4,446), Journal of Personality Assessment (fator de impacto igual a 1,678), International Journal of Offender Therapy and Comparative Criminology (fator de impacto igual a 0,716), Aggression and Violent Behavior (fator de impacto igual a 1,618), entre outros. Por último, recorreu-se a livros especializados no assunto como o Handbook of Psychopathy.

\section{O percurso inicial do Manual Diagnóstico e Estatístico dos Transtornos Mentais}

A Associação Americana de Psiquiatria surgiu em 1840 e era originalmente conhecida como Associação Médica de Superintendentes de Instituições Americanas para Insanos (AMSAI). A partir de então organizou encontros anuais para debater os critérios mais precisos no reconhecimento dos sintomas e quadros nosológicos ${ }^{18}$. Posteriormente, a AMSAI passou a se chamar simplesmente de Associação Médica e Psicológica (MPA).

Em 1913, o Dr. James May, um dos associados da MPA, já havia desenvolvido um sistema uniforme de classificação dos transtornos mentais. Desse modo, a Academia de Medicina de Nova York (NYAM) e a MPA revisaram, em 1933, as nomenclaturas adotadas pelos sistemas norte-americanos de saúde mental, o que culminou no DSM. De 1933 a 1949, o DSM passou por inúmeras reformulações até que a sua primeira edição foi publicada em $1952^{19}$.

\section{O percurso do Manual Diagnóstico e Estatístico dos Transtornos Mentais para o reconhecimento das condições patológicas da personalidade}

À medida que as categorias diagnósticas eram sistematizadas pela APA, formas de classificação dos transtornos mentais foram necessárias para tornar seu reconhecimento mais fácil. Os transtornos de personalidade, por exemplo, são definidos como:

“(...) padrões persistentes no modo de perceber, relacionar-se e pensar sobre o ambiente e sobre si mesmo exibidos em uma ampla faixa de contextos sociais e pessoais. Apenas quando são inflexíveis e mal-adaptativos e causam prejuízo funcional ou sofrimento subjetivo significativo, os traços de personalidade configuram um Transtorno de Personalidade" (20:524).

Dessa forma, os transtornos de personalidade foram caracterizados por um padrão persistente e inflexível que causa sofrimento subjetivo, prejuízo funcional e social e que não pode ser explicado como consequência de outros transtornos mentais ou efeitos fisiológicos determinados por substâncias "estranhas"18. Essa é a concepção atual da Associação Psiquiátrica sobre tais condições clínicas. Entretanto, os gru- pos ou eixos patológicos assim como os critérios para diagnosticá-los modificaram-se ao longo da história do manual.

A primeira edição do DSM na década de 1950 agrupava as doenças mentais em diferentes eixos (ver Tabela 1 para mais detalhes) e ordenava os distúrbios de personalidade da seguinte forma: Padrão de Perturbação da Personalidade; Perturbação dos Traços de Personalidade; Perturbação Sociopática da Personalidade; Sintomas de Reação e Perturbações Transitórias de Personalidade ${ }^{19}$.

A Perturbação Sociopática da Personalidade (320.7), ou Sociopatia, aproximava-se do diagnóstico de Personalidade Psicopática desenvolvido por Kraepelin e Schneider e, por esse motivo, compunha praticamente a mesma classificação para o Transtorno de Personalidade Antissocial' ${ }^{16}$. A Sociopatia era subdividida em Reação Antissocial (320.4), Reação Dissocial (320.5), Desvio Sexual (320.6) e Vícios, e este, por sua vez, subdividia-se em Alcoolismo (322.1) e Drogadição (323).

A APA definiu a Perturbação Sociopática da Personalidade como:

"(...) indivíduos cronicamente anti-sociais que constantemente causavam problemas e seriam incapazes de aprender com os erros, com punições sociais e não seriam leais com pessoas, grupos ou normas. Eles eram frequentemente insensíveis, hedonistas, emocionalmente imaturos, irresponsáveis, com fraco juízo crítico e racionalização dos seus comportamentos" (19:38).

Observa-se que a Sociopatia se referia mais a uma classe de comportamentos do que traços psicológicos. Apesar de as duas primeiras versões do DSM apresentarem uma inegável influência Psicanalítica - um exemplo disso é o uso do termo "traço" para se referir aos padrões de personalidade - a APA preocupou-se primordialmente em compreender como os fatores culturais afetariam e/ou formariam uma pessoa sociopata. Tratava-se da influência do espírito conductualista de Skinner que exercera forte impacto sobre os conhecimentos pedagógico, psicológico e psiquiátrico na década dos anos 1950 e 196021.

Os processos internos, tão enfatizados pelos autores anteriores ao DSM, perderam um pouco de sua força com a influência do Behaviorismo Radical. A APA passou a priorizar a compreensão das variáveis ambientais responsáveis pelas contingências desses conjuntos de comportamentos do que investigar os traços de personalidade subjacentes aos transtornos ${ }^{22}$. Isso pode ser percebido nos cinco subtipos adotados (ver Tabela 1) relacionados aos transtornos de personalidade.

Como passo seguinte, foi publicado em 1968 o DSM-II num esforço da APA em ajustar seus critérios diagnósticos aos da $\mathrm{OMS}^{18}$. Nessa ocasião, a Associação se preocupou em descrever as desordens da personalidade unicamente em termos de traços psicológicos, superando a abordagem condutual então presente no DSM-I. 
Tabela 1. Transtornos de personalidade do DSM-I

\begin{tabular}{|c|c|}
\hline \multicolumn{2}{|l|}{ Distúrbios de personalidade } \\
\hline Padrão de perturbação da personalidade & $\begin{array}{l}\text { Personalidade inadequada } \\
\text { Personalidade esquizoide } \\
\text { Personalidade ciclotímica } \\
\text { Personalidade paranoide }\end{array}$ \\
\hline Perturbação dos traços de personalidade & $\begin{array}{l}\text { Personalidade emocionalmente instável } \\
\text { Personalidade passivo-agressiva } \\
\text { Personalidade compulsiva }\end{array}$ \\
\hline $\begin{array}{l}\text { Perturbação sociopática da } \\
\text { personalidade }\end{array}$ & $\begin{array}{l}\text { Reação antissocial } \\
\text { Reação dissocial } \\
\text { Desvio sexual } \\
\text { Vício } \\
\text { Alcoolismo } \\
\text { Drogadição }\end{array}$ \\
\hline Sintomas de reação & $\begin{array}{l}\text { Distúrbios de aprendizagem } \\
\text { Distúrbios da fala } \\
\text { Enurese } \\
\text { Sonambulismo } \\
\text { Outros sem especificação }\end{array}$ \\
\hline Perturbações transitórias de personalidade & $\begin{array}{l}\text { Reação agressiva de estresse } \\
\text { Reação situacional adulta } \\
\text { Reação de ajustamento do desenvolvi- } \\
\text { mento inicial } \\
\text { Reação de ajustamento na infância } \\
\text { Perturbações do hábito } \\
\text { Perturbações da conduta } \\
\text { Traços neuróticos } \\
\text { Reação de ajustamento na adolescência } \\
\text { Reação de ajustamento na velhice }\end{array}$ \\
\hline
\end{tabular}

Fonte: Adaptado do Manual Diagnóstico e Estatístico de Transtornos Mentais (APA, 1952).

Nessa segunda edição do manual, criaram-se doze categorias diferentes para designar os transtornos de personalidade e outros transtornos não psicóticos (Tabela 2). É nessa edição que surgiram os critérios para a classificação do TPAS assim como o termo PP, por influência da obra de Cleckley ${ }^{1,23-25}$. O TPAS era caracterizado pelos seguintes sintomas: 1) indivíduos não socializados; 2 ) impulsivos; 3 ) sem culpa ou remorso; 4) egoístas; 5) indivíduos insensíveis que racionalizavam seu comportamento e 6) que não aprendiam com a experiência ${ }^{24}$. No entanto, essas descrições a partir da observação clínica não serviam para diagnosticar especificamente a PP, tal como fora descrita por Cleckley, uma vez que poderiam ser aplicadas a outros quadros clínicos ${ }^{26}$. As edições seguintes do manual, de certo modo, foram bem sucedidas no estabelecimento de critérios mais adequados para classificar o TPAS ${ }^{16}$.

A terceira edição do DSM foi publicada em 1980 e se diferencia das anteriores em dois grandes aspectos. O primeiro aspecto é que o DSM-III passa a ser sistematizado em distintas condições clínicas, isto quer dizer que os grupos clínicos são organizados em eixos (avaliação axial) e os diagnósticos passam a ser feitos por uma avaliação semiológica e nosológica mais completa (avaliação multiaxial ou horizontal). Contudo, caso houvesse a coocorrência de mais de uma patologia, predominava aquela considerada a mais profunda e debilitante (avaliação vertical). A reformulação taxonômica
Tabela 2. Transtornos de personalidade do DSM-II

\begin{tabular}{l}
\hline Distúrbios de personalidade \\
\hline 301.0 Paranoide \\
301.1 Ciclotímica ou do humor \\
301.2 Esquizoide \\
301.3 Explosiva \\
301.4 Anancástica ou obsessivo-compulsiva \\
301.5 Histérica \\
301.6 Astênica \\
301.7 Antissocial \\
301.81 Passivo-agressiva \\
301.82 Inadequada \\
301.89 Outra desordem de personalidade específica \\
301.9 Outra desordem de personalidade não específica \\
\hline Fonte: Adaptado do Manual Diagnóstico e Estatístico de Transtornos Mentais (APA, 1968).
\end{tabular}

foi o segundo aspecto alterado na terceira edição do manual. Esses fatos fizeram do DSM-III o mais revolucionário entre todos os manuais da APA até então ${ }^{27}$.

O DSM-III passou a ser dividido em cinco grandes eixos, que englobam diferentes aspectos taxonômicos no que diz respeito à origem dos distúrbios mentais. O Eixo l é composto por transtornos clínicos como, por exemplo, depressão, ansiedade, fobias, entre outros; no Eixo II estão os transtornos do desenvolvimento como as síndromes autísticas e de Down e os transtornos de personalidade; no Eixo III, estão as condições clínicas médicas que podem levar a um distúrbio mental como Alzheimer e mal de Parkinson; o Eixo IV identifica os estressores psicossociais e ambientais como, por exemplo, problemas ocupacionais, econômicos e de moradia; e o Eixo $V$ é composto por uma escala de Avaliação Global do Funcionamento (AGF), dividida em três níveis contínuos (superior, médio e grave) que contém itens numerados de zero a 100 .

A mudança na linguagem utilizada nos manuais da APA, baseada em conceitos advindos da Psicanálise, constitui outro diferencial do DSM-III em relação às duas edições anteriores. Essa nova edição passou a ser um sistema diagnóstico ateórico e que se reportava a dados epidemiológicos e estatísticos, fato que motivou a adoção de outras nomenclaturas. O modelo psicanalítico foi substituído, entre outras razões, por apresentar categorias diagnósticas muito amplas $s^{4,25,28}$. Assim, termos psicanalíticos cederam seu lugar a manifestações clínicas e descrições comportamentais mais objetivas. Por exemplo, "neurose", "histeria", "neurose de angústia" e "melancolia", como utilizadas por Freud, foram substituídas por outras categorias como transtornos depressivos e ansiosos.

As patologias da personalidade, em vez de serem listadas como uma única classe de sofrimentos mentais, foram divididas no DSM-III em três distintos agrupamentos ou clusters. O Grupo A foi resumidamente conhecido como grupo dos excêntricos e nele se encontravam os Transtornos de Personalidade Paranoide, Esquizoide e Esquizotípica. No Grupo B, conhecido como grupo dos impulsivos, imprevisíveis e erráticos, eram encontrados os TPAS, Borderline, Histri- 
ônica e Narcisista. Por fim, no Grupo C, chamado por grupo dos evitativos e ansiosos, encontravam-se os Transtornos de Personalidade de Esquiva, Dependente, Anancástica e Passivo-Agressiva (Tabela 3). O TPAS permaneceu no DSM-III e era definido pelos seguintes sintomas: 1) violação das normas sociais; 2) mentira; 3) roubo; 4) preguiça; 5) não se fixar em um emprego e 6) narcotráfico 1,10,29.

Em 1987, o DSM-III foi revisado e passou a se chamar DSM-III-R $\mathrm{R}^{30}$. A divisão em cinco eixos permaneceu e a semiologia ficou praticamente a mesma. A mudança mais significativa que ocorreu nessa versão do manual foi o surgimento do termo comorbidade ${ }^{16,27}$.

Tabela 3. Transtornos de personalidade do DSM-III

\begin{tabular}{lll}
\hline & \multicolumn{2}{c}{ Transtornos do desenvolvimento } \\
& Distúrbios de personalidade (Eixo II) \\
\hline A & B & C \\
(Excêntricos) & $\begin{array}{l}\text { (Impulsivos, erráticose } \\
\text { imprevisíveis) }\end{array}$ & (Ansiosos) \\
Paranoide & Antissocial & Evitativo \\
Esquizoide & Borderline & Dependente \\
Esquizotípico & Histriônico & Anancástico \\
& Narcisisto & Passivo-agressivo \\
& Sádico & Depressivo-distímico \\
\hline
\end{tabular}

Fonte: Adaptado do Manual Diagnóstico e Estatístico de Transtornos Mentais (APA, 1980).

No DSM-III, se um indivíduo fosse diagnosticado com mais de uma condição nosológica, prevaleceria aquela considerada mais grave e profunda. Esse modo de classificar os transtornos é conhecido como verticalização diagnóstica. Contudo, no DSM-III-R, a APA pressupunha que, se um mesmo indivíduo portasse mais de uma condição diagnóstica, não deveria prevalecer uma condição em relação à outra, pois ambas trariam algum grau de comprometimento funcional ao indivíduo. Dessa forma, a prevalência de duas ou mais categorias diagnósticas passou a ser conhecida como comorbidade, um dos conceitos mais importantes originados na Psiquiatria para a pesquisa e a prática clínica 27,31.

Outra mudança a ser notada no DSM-III-R refere-se aos transtornos de personalidade. Duas taxonomias saíram da classificação dos Transtornos de Personalidade (Tabela 4), a Personalidade Sádica (Grupo B) e a Personalidade Depressiva (Grupo C), por não apresentarem suporte empírico que as mantivesse ali. Tanto o Transtorno Sádico quanto o Depressivo moveram-se para o Eixo I. O Sadismo foi incluído entre as parafilias, apesar de, em alguns casos, estar correlacionado à conduta antissocial e à PP (1), e a Personalidade Depressiva foi incluída no espectro dos transtornos do humor.

Em 1994, foi editado o quarto Manual Diagnóstico e Estatístico de Doenças Mentais, o DSM-IV²0. Essa quarta versão do manual é dividida da mesma forma que o DSM-III e o DSMIII-R. No entanto, algumas modificações foram percebidas nessa edição. Em vez de meramente descrever e agrupar os sintomas, a APA acrescentou alguns itens antes de deduzir os respectivos critérios semiológicos, entre eles características e transtornos associados, características específicas à cultura, à idade e ao gênero, prevalência, padrão familiar e diagnóstico diferencial.

Os transtornos do Eixo II (transtornos do desenvolvimento), especificamente os de personalidade, sofreram uma pequena alteração (Tabela 5). O Transtorno Passivo-Agressivo de Personalidade saiu dessa classificação no DSM-IV, pelo mesmo motivo que as personalidades sádica e depressiva saíram do DSM-III-R.

Tabela 4. Transtornos de personalidade do DSM-III-R

\begin{tabular}{lll}
\hline & \multicolumn{2}{c}{$\begin{array}{c}\text { Transtornos do desenvolvimento } \\
\text { Distúrbios de personalidade (Eixo II) }\end{array}$} \\
\hline $\begin{array}{l}\text { A } \\
\text { (Excêntricos) }\end{array}$ & $\begin{array}{l}\text { B } \\
\text { (Impulsivos, erráticose } \\
\text { imprevisíveis) }\end{array}$ & $\begin{array}{l}\text { (Ansiosos) } \\
\text { Paranoide }\end{array}$ \\
Esquizoide & Antissocial & Evitativo \\
Esquizotípico & Borderline & Dependente \\
& Histriônico & Anancástico \\
\hline
\end{tabular}

Fonte: Adaptado do Manual Diagnóstico e Estatístico de Transtornos Mentais (APA, 1987).

Tabela 5. Transtornos de personalidade do DSM-IV

\begin{tabular}{lll}
\hline & \multicolumn{2}{c}{$\begin{array}{c}\text { Transtornos do desenvolvimento } \\
\text { Distúrbios de personalidade (Eixo II) }\end{array}$} \\
\hline $\begin{array}{l}\text { A } \\
\text { (Excêntricos) }\end{array}$ & $\begin{array}{l}\text { (Impulsivos, erráticos e } \\
\text { imprevisíveis) }\end{array}$ & (Ansiosos) \\
Paranoide (301.0) & Antissocial (301.7) & Esquivo (301.82) \\
Esquizoide (301.20) & Borderline (301.83) & Dependente (301.6) \\
Esquizotípico (301.22) & Histriônico (301.50) & Anancástico (301.4) \\
& Narcisisto (301.81) & \\
\hline
\end{tabular}

Fonte: Adaptado do Manual Diagnóstico e Estatístico de Transtornos Mentais (APA, 1994).

A APA, na quarta edição, manteve a nomenclatura Transtorno de Personalidade Antissocial definindo-a como:

“(...) um padrão invasivo de desrespeito e violação dos direitos dos outros, que inicia na infância ou começo da adolescência e continua na idade adulta. Este padrão também é conhecido como Psicopatia, Sociopatia ou Transtorno de Personalidade Dissocial" (20:608).

As características desse transtorno e de quadros clínicos associados se assemelham, em algum nível, às utiliza- 
das pelo criador do PCL-R (Psychopathy Checklist Revised), desenvolvidas por Robert Hare para o diagnóstico da PP'16. Enaltecimento de si mesmo, arrogância, insensibilidade, desprezo pelos sentimentos alheios, cinismo, mentira e encanto superficial estão entre os critérios que se associam adequadamente na descrição do primeiro fator (Déficits Afetivos) do $P C L-R^{32}$. Todavia esses sintomas não estão listados adequadamente nos critérios de diagnóstico do TPAS (Tabela 6).

O DSM-IV descreve o TPAS como mais frequentemente encontrado em contextos urbanos e está geralmente relacionado a um baixo nível socioeconômico. É mais frequente em homens que em mulheres numa proporção de três para

Tabela 6. Critérios de classificação do transtorno de personalidade antissocial DSM-IV

\begin{tabular}{l}
\hline Critérios diagnósticos do transtorno de personalidade antissocial \\
\hline (301.7) \\
(A) Padrão invasivo de desrespeito e violação dos direitos dos outros, que ocorre desde os \\
15 anos, como indicado por pelo menos três dos seguintes critérios: \\
- Fracasso em conformar-se às normas sociais com relação a comportamentos legais, \\
indicados pela execução repetida de atos que constituem motivo de detenção \\
- Propensão para enganar, indicada por mentir repetidamente, usar nomes falsos ou \\
ludibriar os outros para obter vantagens pessoais ou prazer \\
- Impulsividade ou fracasso em fazer planos para o futuro \\
- Irritabilidade e agressividade, indicadas por repetidas lutas corporais ou agressões \\
físicas \\
- Desrespeito irresponsável pela segurança pública ou alheia \\
- Irresponsabilidade consistente, indicada por um repetido fracasso em manter um \\
comportamento laboral consistente ou honrar obrigações financeiras \\
- Ausência de remorso, indicada por indiferença ou racionalização por ter ferido, maltra- \\
tado ou roubado outra pessoa
\end{tabular}

(B) 0 indivíduo tem, no mínimo, 18 anos de idade

(C) Existem evidências de transtorno de conduta com início antes dos 15 anos: (1) Agressão a outras pessoas e animais

Frequentemente brigava, ameaçava ou intimidava os outros

Frequentemente iniciava embates corporais

Costumava usar armas que poderiam causar sérios danos aos outros (tacos, estilhaços de garrafas, facas e outras armas)

Era cruel com as pessoas

Era cruel com os animais

Roubava enquanto confrontava uma vítima (assalto, extorção, roubo à mão armada e furto)

Forçava alguém em ter relações sexuais

\section{(2) Destruição de propriedade}

Engajava-se deliberadamente em botar fogo com a intenção de causar sérios danos Quebrava ou destruía deliberadamente bem alheios, tais como carros, casas ou prédios (por outro modo que não seja botar fogo)

Frequentemente mentia para obter favores dos outros para evitar as obrigações (enganava os outros)

Roubava coisas sem valor sem confrontar a vítima (lojas de departamento, mas sem invadir e falsificar documentos)

\section{(3) Sérias violações às regras}

Frequentemente saía de casa à noite sem permissão dos pais, antes dos 13 anos Fugia de casa por toda a noite, deixando pelo menos duas vezes a casa dos pais ou dos responsáveis (ou pelo menos uma vez sem retornar por um longo período)

Frequentemente fugia da escola, antes dos 13 anos $\mathrm{e}$

(D) A ocorrência do comportamento antissocial não se dá exclusivamente durante o curso de esquizofrenia ou episódio maníaco

Fonte: Adaptado da American Psychiatric Association (1994). um (3:1) em amostras comunitárias. Em contextos clínicos, a frequência de manifestação do TPAS aumenta de 3\% a 30\%, tanto para homens quanto para mulheres, elevando-se em até $60 \%$ dos casos em contextos forenses ${ }^{20}$.

O TPAS pode estar associado a outros transtornos de personalidade, porém existem diferenças entre aquela condição clínica e os outros distúrbios. Por exemplo, são características bem marcantes e definitivas no TPAS os comportamentos criminosos, violência, manipulação para obter vantagens para si e história de problemas de conduta com início na infância e na adolescência. Os critérios diagnósticos para essa condição clínica podem ser observados na tabela 6 .

Por fim, a APA lançou, no ano de $2000^{33}$, a revisão do quarto Manual Diagnóstico e Estatístico de Doenças Mentais, abreviada como DSM-IV-TR*. Essa versão do manual estabelece cinco tipos de transtornos de personalidade de acordo com Widiger ${ }^{16}$ : o Lábil, o Desinibido, o Apático, o Paranoide e o Agressivo - que se referem quase que exclusivamente ao TPAS. No entanto, os diagnósticos específicos de transtornos de personalidade permanecem os mesmos publicados no DSM-IV (Tabela 5).

\section{DISCUSSÃO}

\section{Algumas considerações sobre o DSM como sistema diagnóstico categorial}

O DSM é amplamente utilizado por profissionais da área de saúde mental, pelo seu mérito de continuamente procurar uma linguagem objetiva e clara na comunicação dos seus critérios diagnósticos.

Termos como neurose e histeria saíram definitivamente do sistema de classificação da APA, evitando, desse modo, que os diagnósticos fossem utilizados de forma pejorativa.

Deve-se reconhecer que a descrição dos quadros clínicos, especialmente dos transtornos de personalidade, inspirou uma série de pesquisas desde o primeiro manual, o DSM-I, as quais resultaram no avanço de áreas como a Psicologia Clínica e a Farmacologia27. Esse avanço, por sua vez, propiciou ajuste psicossocial e melhora na qualidade de vida de indivíduos portadores de algum transtorno mental. Veja a tabela 7 para observar o panorama geral de desenvolvimento dos DSM.

Embora de grande utilidade entre os profissionais da área, os manuais psiquiátricos, entretanto, são muito gerais em suas descrições e excluem uma quantidade significativa de informações como origem, desenvolvimento, estrutura, entre outras ${ }^{34}$. Isso é notório no TPAS, uma vez que o DMS enfatiza minimamente as dimensões subjacentes à personalidade antissocial, privilegiando as descrições sintomáticas ${ }^{35-37}$.

* A sigla TR significa Text Revision em inglês, termo traduzido livremente para o português como Revisão de Texto. 
Tabela 7. Contexto histórico do estudo da personalidade antissocial - Sistema Categorial (DSM)

\begin{tabular}{|c|c|c|c|c|c|}
\hline Data & Fonte & Nomenclatura & Descrição & Percepção social & Prognóstico percebido \\
\hline 1952 & $\begin{array}{l}\text { DSM (APA-American } \\
\text { Psychiatric Association) }\end{array}$ & $\begin{array}{l}\text { Transtorno Sociopático de } \\
\text { Personalidade } \\
\text { (Reação Antissocial } \\
\text { Reação Dissocial } \\
\text { Desvio Sexual e Vício) }\end{array}$ & $\begin{array}{l}\text { Apesar da influência Psica- } \\
\text { nalítica, está mais orientado } \\
\text { a reconhecer as perspectivas } \\
\text { sociais como origem dos com- } \\
\text { portamentos criminosos }\end{array}$ & Depreciativa & $\begin{array}{l}\text { Capacidade de recuperação muito } \\
\text { pobre }\end{array}$ \\
\hline 1968 & DSM-II & $\begin{array}{l}\text { Transtorno de Personalidade } \\
\text { Antissocial (Dissocial e Sociopa- } \\
\text { ta saem da classificação) }\end{array}$ & $\begin{array}{l}\text { Focado nos traços de personali- } \\
\text { dade do Psicopata } \\
\text { Alguns dos critérios de Cleckley }\end{array}$ & Depreciativa & $\begin{array}{l}\text { Capacidade de recuperação muito } \\
\text { pobre }\end{array}$ \\
\hline 1980 & DSM-III & $\begin{array}{l}\text { Transtorno de Personalidade } \\
\text { Antissocial }\end{array}$ & $\begin{array}{l}\text { Ateórico. Violaçãa crônica das } \\
\text { regras sociais: transtornos de } \\
\text { conduta }\end{array}$ & $\begin{array}{l}\text { Depreciativa } \\
\text { Inclui a maioria dos ofensores }\end{array}$ & $\begin{array}{l}\text { Capacidade de recuperação muito } \\
\text { pobre, mas os sintomas diminuem } \\
\text { com a idade }\end{array}$ \\
\hline 1987 & DSM-III-R & $\begin{array}{l}\text { Transtorno de Personalidade } \\
\text { Antissocial }\end{array}$ & $\begin{array}{l}\text { Ateórico. Transtorno de Persona- } \\
\text { lidade Antissocial }\end{array}$ & $\begin{array}{l}\text { Violação crônica das regras } \\
\text { sociais: transtornos de conduta }\end{array}$ & $\begin{array}{l}\text { Depreciativa } \\
\text { Inclui a maioria dos ofensores }\end{array}$ \\
\hline 1994 & DSM-IV & $\begin{array}{l}\text { Transtorno de Personalidade } \\
\text { Antissocial }\end{array}$ & $\begin{array}{l}\text { Ateórico. Focado em critérios } \\
\text { categoriais/comportamentais }\end{array}$ & $\begin{array}{l}\text { Depreciativa e equiparada à } \\
\text { condenação da justiça }\end{array}$ & $\begin{array}{l}\text { Capacidade de recuperação muito } \\
\text { pobre, mas os sintomas diminuem } \\
\text { com a idade }\end{array}$ \\
\hline 2000 & DSM-IV-TR & $\begin{array}{l}\text { Transtorno de Personalidade } \\
\text { Antissocial }\end{array}$ & $\begin{array}{l}\text { Ateórico. Focado em critérios } \\
\text { categoriais/comportamentais e } \\
\text { faz parte do subtipo Agressivo }\end{array}$ & $\begin{array}{l}\text { Depreciativa e equiparada à } \\
\text { condenação da justiça }\end{array}$ & $\begin{array}{l}\text { Capacidade de recuperação muito } \\
\text { pobre, mas os sintomas diminuem } \\
\text { com a idade }\end{array}$ \\
\hline
\end{tabular}

Fonte: Adaptado de Arrigo e Shipley (2001) com a permissão da SAGE Publications of International Journal of Offender Therapy and Comparative Criminology e desenvolvido por Alvarenga em sua dissertação de mestrado.

A limitação de informação nos manuais DSM parece estar associada ao fato de muitos profissionais da saúde utilizarem o sistema de classificação de forma inflacionada, exagerada. Assim, confecciona-se uma quantidade de diagnósticos associados - comorbidade intra e intereixos -, provavelmente inexistentes, o que termina por prejudicar as diretrizes de tratamento em vez de auxiliá-las. Autores como Matos et al. ${ }^{27}$ ressaltam outra dificuldade no manejo do DSM: a crença de infalibilidade e exatidão do sistema de classificação proposto pelo manual. Essa ideia prejudica, inclusive, uma das propostas iniciais da APA que seria a de gerar pesquisas acerca de seus critérios diagnósticos para torná-los mais precisos. O que inicialmente deveria ser uma linguagem consensual passou a ser um problema pela restrição e falta de precisão para reconhecer diferentes condições nosológicas ou categorias $^{4,2,26}$.

No que se refere aos distúrbios de personalidade e, mais especialmente do TPAS, o acometimento da dificuldade de julgamento clínico pode estar referendado pela definição dada pelo DSM. Por exemplo, o TPAS é definido como um "transtorno de personalidade composto por comportamentos observáveis e traços de personalidade inferidose depreciados pela sociedade ${ }^{\prime 34}$. Cabe se perguntar que traços seriam esses?

Outro problema referente à classificação do TAPS é que, no contexto clínico e forense, os sintomas descritos desse quadro são geralmente empregados como sinônimos de Psicopatia, Sociopatia e Transtorno Dissocial. No entanto, há divergências no que se refere à definição desses termos ${ }^{13,15,16}$. O sistema categorial de avaliação dos DSM pressupõe uni- dades de observação fechadas e predefinidas a partir de deduções clínicas empíricas ${ }^{36,38-40}$. Estas últimas, por sua vez, multiplicam-se graças ao surgimento de diagnósticos de comorbidade, que alimentam posteriormente os futuros DSM. Enquanto isso, informações-base como a natureza e a estrutura das doenças mentais permanecem escuras. Uma das discussões para o DSM-V deve ir além das descrições categóricas e compreender as variações muito intensas existentes em certos traços que apresentam relevância clínica para o diagnóstico e tratamento, pois abordagens psiquiátricas baseadas em observações clínicas, buscando a relação e a organização entre os sintomas manifestados na composição do diagnóstico, formam a maioria entre os modelos desenvolvidos para compreender e definir o funcionamento do TPAS 16,17,40-42. Por esse motivo, é importante, para se fazer uma revolução tão grande quanto a vista na publicação do DSM-III, pensar como a integração do sistema dimensional será implementada na nova versão do DSM, uma vez que ainda não está claro que espectros de traços serão determinados e a reflexão de suas respectivas dimensões (intensidade) a pontos que sejam considerados necessariamente patológicos ${ }^{17,38,39,41,42}$.

Por esse motivo, as medidas ou os testes de personalidade vêm sendo desenvolvidos. Dessa forma, eles se tornam instrumentos promissores para a avaliação de quadros patológicos da personalidade. Os testes de personalidade vêm sendo desenvolvidos a partir da análise fatorial de testes sensíveis a padrões afetivos, cognitivos e comportamentais ${ }^{42,43}$. Os modelos fatoriais buscam, de maneira matemática, encontrar e organizar com relativa independência grupos ou traços que permitam avaliar como se dispõe a estrutura da 
personalidade ${ }^{44}$. Vista a importância da dimensionalidade e a utilidade de sua aplicação bem como a descrição de traços para a avaliação do TPAS teria como o modelo prototípico existente no PCL-R $\mathrm{R}^{17,38,40,45}$.

Uma forma de melhorar tal panorama talvez seja a de apostar pela investigação em nível dimensional, em que as classificações - e as categorias que as descrevem - sejam submetidas a um processo de investigação psicológica diferencial. Essa investigação seria nos três níveis de análise comportamental: fatorial, neuropsicológica e cognitiva. As investigações com a utilização do PCL-R é um bom exemplo de como a perspectiva dimensional poderia complementar a compreensão de distúrbios da personalidade e ajudar na categorização psiquiátrica ${ }^{38}$.

O PCL-R apresenta basicamente duas grandes dimensões, o comprometimento afetivo que é apoiado por alguns traços (mentira, superestima, manipulação, frieza, ausência de culpa e falta de empatia) e os desvios de condutas apontadas pela manifestação de comportamentos socialmente desadaptativos - agressividade, impulsividade, tendência ao tédio, estilo parasitário e cometem e reincidem em diferentes de tipos de infrações $6,15,32,40,44,46$. Neste sentido, o PCL-R permite inferir a dimensionalidade de uma série de traços e comportamentos desviantes e a melhor forma de avaliá-los, uma vez que os agrupam para serem reconhecidos como uma categoria, no caso, o TPAS com um prejuízo mais acentuado, como também permite identificar o nível de comprometimento da personalidade e seu impacto social - a relação com a quantidade de crimes cometidos ${ }^{38}$.

Segundo aquelas investigações, a psicopatia não seria uma condição que aparece na idade adulta, que algumas pessoas a possuem e outras não. A psicopatia seria uma dimensão psicológica em que as pessoas a apresentam em um continuum, a maioria menos e uma minoria em excesso ou com quase total ausência. O retardo mental é talvez o único transtorno dos DSM que incorpora o critério dimensional. Sem um ponto de corte de desempenho cognitivo, o retardo mental dificilmente é diagnosticado. O mesmo poderia ocorrer com os demais transtornos mentais.

\section{CONCLUSÃO}

O DSM tornou-se uma referência internacional para o diagnóstico de várias desordens mentais e entre elas estão os transtornos de personalidade. A Personalidade Antissocial é um desses transtornos que têm recebido grande atenção da comunidade científica pelo incontestável prejuízo que causa à sociedade, de forma geral, e às pessoas. Entretanto, o DSM é passível de algumas críticas no que se refere à escolha de seus métodos de classificação desse distúrbio. O sistema categórico para a avaliação do TPAS perde em relação a outros procedimentos diagnósticos por não identificar outras variáveis associadas à sua manifestação, por exemplo, componentes genéticos e neurológicos, fatores relacionados à origem e ao desenvolvimento ${ }^{47}$, bem como o tipo de crime, o tratamento e a reincidência criminal ${ }^{6,10}$.

Bornstein $^{48}$, Bernstein et al. ${ }^{49}$ e Widiger e Clark ${ }^{25}$ acreditam que as atuais críticas sobre o modelo encontrado no DSM-IV e no DSM-IV-TR contribuirão, sem dúvida alguma, para a reformulação do próximo manual, o DSM-V. De qualquer forma, sabe-se que o sistema categórico não pretende fazer uma avaliação global do transtorno de personalidade, quer seja do Antissocial ou não, tampouco o sistema categórico deve ser abandonado ${ }^{17}$. O que os manuais procuram é desenvolver, basicamente, uma linguagem objetiva e acessível e não um sistema final de reconhecimento de manifestações patológicas. Contudo, suas limitações instigaram outras formas mais fidedignas para avaliar esses construtos em diferentes contextos acadêmicos e forenses, como o sistema de avaliação dimensional ${ }^{15}$. Nesse sentido, há muitas expectativas para o futuro DSM-V, entre elas a incorporação de informações advindas das investigações dimensionais, com isso desenvolvendo um modelo que permite uma avaliação de traços contínuos em vez de um reconhecimento puramente discreto ${ }^{37}$ como acontece com a PP avaliada pelo PCL- $\mathrm{R}^{6}$.

\section{REFERÊNCIAS}

1. Arrigo BA, Shipley $S$. The confusion over psychopathy (I): historical considerations. Inter J Offender Ther Comp Criminol. 2001;45(3):325-44.

2. Collier B. The Candlelight Project. Biopsychiatry Illuminated, 58, 2003. Disponível em: http://adhd-report.com/biopsychiatry/bio_10.html. Acessado em: 27/6/2006.

3. Wilson NJ. The Utility of the Psychopathy Checklist-Screening Version (PCL-SV) for Predicting Serious Violent Recidivism in a New Zealand Offender Sample. Doctorate of Philosophy at the University of Waikato, New Zealand; 2003. p. 313.

4. Jablensky A. The nature of psychiatric classification: issues beyond ICD-10 and DSM-IV. Aust N Z J Psychiatry. 1999;33:137-44.

5. Kendell $R$, Jablensky A. Distinguishing between the validity and utility of psychiatric diagnoses. Am J Psychiatry. 2003;160:4-12.

6. Hare RD, Neumann CS. Psychopathy as a clinical and empirical construct. Annu Rev Clin Psychol. 2008:4:217-46.

7. Organização Mundial da Saúde (OMS). CID-10: Classificação de Transtornos Mentais e de Comportamento: Descrições Clínicas e Diretrizes Diagnósticas. Porto Alegre: Editora Artes Médicas Sul Ltda., 1993.

8. American Psychiatric Association (APA). Psychiatric Diagnosis and the Diagnostic Statistical Manual of Mental Disorders (Fourth Edition - DSM-IV). Fact Sheet. 1997;1-4.

9. Colorado Departament of Public Health and Enviroment (CDPHE). New International Classification of Diseases (ICD-10): The History and Impact. Brief Health Statistics Section of CDPHE. 2001;21:1-4. Disponível em: http://www.cdphe.state.co.us/hs/. Acessado em: 20/9/2008.

10. Chapman AL, Gremore TM, Farmer RF. Psychometric analysis of the Psychopathic Personality Inventory (PPI) with female inmates. J Pers Assess. 2003;80:164-72.

11. Hare RD. Psychopathy and antisocial personality disorder: a case of diagnostic confusion. Psychiatr Times. 1996;13:1-8.

12. Hare RD, Hart SD, Harpur T. Psychopathy and the DSM-IV Criteria for Antisocial Personality Disorder (Diagnostic Issues in DSM-IV). J Abnorm Psychol. 1991;100(3):391-8.

13. Shipley S, Arrigo BA. The confusion over psychopathy (II): implications for forensic (correctional) practice. Inter J Offender Ther Comp Criminol. 2001;45(4):407-20. 
14. Skeem JL, Poythress NG, Edens JF, Lilienfeld SO, Cale EM. Psychopathic personality or personalities? Exploring potential variants of psychopathy and their implications for risk assessment. Aggress Violent Behav. 2003;8:513-46.

15. Flores-Mendoza (E, Alvarenga MAS, Herrero 0, Abad FJ. Factor structure and behavioural correlates of the Psychopathy Checklist-Revised (PCL-R) in a Brazilian prisoner sample. Pers Individ Dif. 2008:45:584-90.

16. Widiger TA. Psychopathy and DSM-IV Psychopathology. In: Patrick CJ, editor. Handbook of Psychopathy. New York: The Guilford Press; 2006. p. 156-71.

17. Brown TA, Barlow DA. Dimensional versus categorical classification of mental disorders in the fifth edition of the Diagnostic and statistical Manual of Mental Disorders and beyond: comment on the special section. J Abnorm Psychol. 2005;114(4):551-6.

18. Ozarin LD. DSM: a brief historial note. Psychiatry news main frame, others features, s/d. Disponível em: http://www.psych.org/pnews/98-04-03/hx.html. Acessado em 21/8/2008.

19. American Psychiatric Association. Diagnostic and Statistical Manual of Mental Disorders. Washington, DC: American Psychiatric Association; 1952.

20. American Psychiatric Association. Diagnostic and Statistical Manual of Mental Disorders. 4. ed. Washington, DC: American Psychiatric Association; 1994.

21. McCord W, McCord J. The psychopathy: an essay on the criminal mind. NJ: Van Nostrand, Princeton; 1964

22. Martens WHJ. Antissocial and psychopathic personality disorders: cause, cure, and remission - A review article. Inter J Offender Ther Comp Criminol. 2000;44(4):406-30

23. Cleckley H. The Mask of Sanity (5th ed.). C. V. Mosby, St. Louis, M0, 1941/1976.

24. American Psychiatric Association. Diagnostic and Statistical Manual of Mental Disorders (Rev. Ed.). Washington, DC: American Psychiatric Association; 1968

25. Widiger T, Clark LA. Toward DSM-V and the classification of psychopathology. Psychol Bull. 2000;126(6):946-63.

26. First M, Spitzer R. The DSM: not perfect, but better than the alternative. Psychiatric Times. 2003;20(4). Disponível em: http://psychiatrictimes.com. Acessado em: 21/8/2006.

27. Matos EG, Matos TMG, Matos GMG. A importância e as limitações do uso do DSM-IV na prática clínica. Rev Psiquiatr Rio Gd Sul. 2005;27(3):312-18.

28. Rodrigues JTA. Medicação como única resposta: uma miragem do contemporâneo. Psicol Estudo. 2003;8(1):13-22.

29. American Psychiatric Association. Diagnostic and Statistical Manual of Mental Disorders. 3. ed. Washington, DC: American Psychiatric Association; 1980.

30. American Psychiatric Association. Diagnostic and Statistical Manual of Mental Disorders. 3. ed. Washington, DC: American Psychiatric Association; 1987.

31. Lewinsohn PM. Comorbidity of Mood and Anxiety Disorders (II). In: Maser JD, Cloninger CR (editors). Washington, DC: American Psychiatric Press; 1990.
32. Hare RD. The Psychopathy Checklist-Revised (PCL-R). USA: Multi Health Systems; 1991.

33. American Psychiatric Association. Diagnostic and Statistical Manual of Mental Disorders. 4 ed. Text Revision). Washington, DC: American Psychiatric Association; 2000.

34. Widiger T, Trull T. Behavioural indicators, hypothetical constructs, and personality disorders. J Pers Disord. 1987;1:82-7.

35. Widiger TA. Mental disorders as discrete clinical conditions: dimensional versus categorical classification. In: Turner SM, Hersen M. (editors.). Adult Psychopathology and Diagnosis 3. ed. 3-23). New York: Wiley; 1997.

36. Brown TA, Barlow DA. Dimensional versus categorical classification of mental disorders in the fifth edition of the Diagnostic and statistical Manual of Mental Disorders and beyond: comment on the special section. J Abnorm Psychol. 2005;114(4):551-56.

37. Spitzer RL, First MB, Shedler J, Westen D, Skodol AE. Clinical utility of five dimensional systems for personality diagnosis a "consumer preference" study. J Nerv Ment Dis. 2008;196(5):356-74.

38. Livesley WJ. A framework for integrating dimensional and categorical classifications of personality disorder. J Pers Disord. 2007;21:199-224.

39. Trull TJ, Tragesser SL, Solhan M, Schwartz-Mette R. Dimensional models of personality disorder: Diagnostic and Statistical Manual of Mental Disorders Fifth Edition and beyond. Curr Opin Psychiatry. 2007;20:52-6.

40. Livesley WJ. Commentary on reconceptualizing personality disorder categories using trait dimensions. J Pers. 2001;69(2):277-86

41. Oldham JM, Skodol AE. Charting the future of Axis II. J Pers Disord. 2000;14:17-29.

42. Morey LC, Hopwood CJ, Gunderson JD, Skodol AE, Shea MT, Yen S, et al. Comparison of alternative models for personality disorders. Psychol Med. 2007;37:983-94.

43. McCrae RR, Costa PTJ. Personality in adulthood. New York: The Guilford Press; 1990.

44. Farmer RF, Nelson-Gray R0. Behavioral assessment of personality disorders. Behav Res Ther. 1999;37:347-68.

45. Hare RD. Psychopathy: a clinical construct whose time has come. Crim Justice Behav. 1996;23:25-54.

46. Hare RD. Without conscience: The disturbing world of the psychopaths among us. New York: The Guilford Press; 1993.

47. Alvarenga MAS, Mansur-Alves M, Franco FM. 0 desenvolvimento das dimensões antissociais da personalidade (231-258). In: Penna FJ, Haase VG (editores.). Aspectos biopsicossociais da saúde na infância e adolescência. Belo Horizonte: Coopmed, 2009.

48. Bornstein RF. Reconceptualizing personality disorder diagnosis in the DSM-V: The discriminant validity change. Clinical Psychology: Science and Practice. 1998;11:69-94.

49. Bernstein DP, Iscan C, Mase J, Links P, Vaglum P. Opinions of personality disorder experts regarding the DSM-IV personality disorders classification system. J Pers Disord. 2007;21(5):536-51. 